\title{
Mapping the Structural Path for Allosteric Inhibition of a Short-Form ATP Phosphoribosyltransferase by Histidine
}

\author{
Catherine M. Thomson, ${ }^{\dagger}$ Magnus S. Alphey, ${ }^{\dagger}$ Gemma Fisher, and Rafael G. da Silva*(i) \\ School of Biology, Biomedical Sciences Research Complex, University of St Andrews, St Andrews, Fife KY16 9ST, U.K. \\ Supporting Information
}

ABSTRACT: ATP phosphoribosyltransferase (ATPPRT) catalyzes the first step of histidine biosynthesis, being allosterically inhibited by the final product of the pathway. Allosteric inhibition of long-form ATPPRTs by histidine has been extensively studied, but inhibition of short-form ATPPRTs is poorly understood. Short-form ATPPRTs are hetero-octamers formed by four catalytic subunits $\left(\mathrm{HisG}_{\mathrm{S}}\right.$ ) and four regulatory subunits (HisZ). $\mathrm{HisG}_{\mathrm{S}}$ alone is catalytically active and insensitive to histidine. HisZ enhances catalysis by $\mathrm{HisG}_{\mathrm{S}}$ in the absence of histidine but mediates allosteric inhibition in its presence. Here, steady-state and pre-steady-state kinetics establish that histidine is a non-

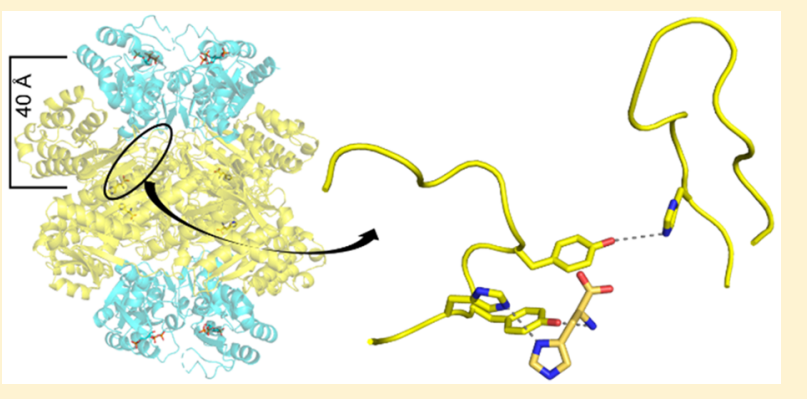
competitive inhibitor of short-form ATPPRT and that inhibition does not occur by dissociating HisG from the hetero-octamer. The crystal structure of ATPPRT in complex with histidine and the substrate 5-phospho- $\alpha$-D-ribosyl-1-pyrophosphate was determined, showing histidine bound solely to HisZ, with four histidine molecules per hetero-octamer. Histidine binding involves the repositioning of two HisZ loops. The histidine-binding loop moves closer to histidine to establish polar contacts. This leads to a hydrogen bond between its Tyr263 and His104 in the Asp101-Leu117 loop. The Asp101-Leu117 loop leads to the HisZ- $-\mathrm{HisG}_{\mathrm{S}}$ interface, and in the absence of histidine, its motion prompts $\mathrm{HisG}_{\mathrm{S}}$ conformational changes responsible for catalytic activation. Following histidine binding, interaction with the histidine-binding loop may prevent the Asp101-Leu117 loop from efficiently sampling conformations conducive to catalytic activation. Tyr263Phe-PaHisZ-containing PaATPPRT, however, is less susceptible though not insensitive to histidine inhibition, suggesting the Tyr263-His104 interaction may be relevant to yet not solely responsible for transmission of the allosteric signal.

$\mathrm{N}$ egative feedback control is a common strategy that evolved in many biosynthetic pathways, where the final biosynthetic product allosterically inhibits the first and often flux-controlling enzyme of the pathway. ${ }^{1,2}$ The biosynthesis of histidine showcases a classical example of negative feedback regulation of biochemical pathways via allosteric inhibition. ${ }^{3}$ The pathway starts with the $\mathrm{Mg}^{2+}$-dependent and reversible nucleophilic attack of adenosine $5^{\prime}$-triphosphate (ATP) on 5phospho- $\alpha$-D-ribosyl-1-pyrophosphate (PRPP) to generate $N^{1}$ (5-phospho- $\beta$-D-ribosyl)-ATP (PRATP) and inorganic pyrophosphate $\left(\mathrm{PP}_{\mathrm{i}}\right)$, catalyzed by the flux-controlling enzyme ATP phosphoribosyltransferase (ATPPRT, EC 2.4.2.17) (Scheme 1). ${ }^{4}$ After eight subsequent reactions, PRATP is converted to L-histidine, which allosterically inhibits ATPPRT. ${ }^{5}$

ATPPRT is a model system for interrogation of the allosteric control of enzyme catalysis, ${ }^{6-11}$ with applications in synthetic biology ${ }^{12,13}$ and antibiotic discovery. ${ }^{14,15}$ Accordingly, allosteric inhibition of ATPPRT by histidine has been extensively investigated with the homohexameric long form of the enzyme $\left(\mathrm{His}_{\mathrm{L}}\right)$, which is present in most histidine-synthesizing organisms. ${ }^{3,9,14,16-18}$ On the other hand, inhibition of shortform ATPPRT, found instead in archaea and some eubacteria, is poorly understood.

Short-form ATPPRTs assemble in a hetero-octamer consisting of four regulatory subunits flanked on each side by a dimer of catalytic subunits. The active site is found solely within $\mathrm{HisG}_{\mathrm{S}}$, a version of $\mathrm{HisG}_{\mathrm{L}}$ in which the C-terminal domain responsible for allosteric inhibition is missing. ${ }^{8,19}$ $\mathrm{His}_{\mathrm{S}}$ on its own forms homodimers with reduced catalytic activity, and it is insensitive to histidine. ${ }^{6,20} \mathrm{HisG}_{\mathrm{S}}$ catalysis is allosterically enhanced in the hetero-octameric ATPPRT holoenzyme by interaction with regulatory protein HisZ, a catalytically inactive paralogue of histidyl-tRNA synthetase (HisRS). ${ }^{8,21}$ In the presence of histidine, however, HisZ mediates the allosteric inhibition of ATPPRT. Only one crystal structure of a short-form ATPPRT in complex with histidine has been published so far, that of the thermophile Thermotoga maritima, where, unexpectedly, eight molecules of histidine per hetero-octamer are found, located at the $\mathrm{HisG}_{\mathrm{S}}-\mathrm{HisZ}$ interface and showing more polar interactions with the catalytic subunit than with the regulatory one. ${ }^{22}$

We have recently reported the structural and kinetic basis for allosteric activation of the hetero-octameric ATPPRT from the psychrophilic bacterium Psychrobacter arcticus (PaATPPRT).$^{10,11,20}$ In the work presented here, we use

Received: April 1, 2019

Revised: June 10, 2019

Published: June 25, 2019 
Scheme 1. ATPPRT-Catalyzed Reaction Is Inhibited by Histidine

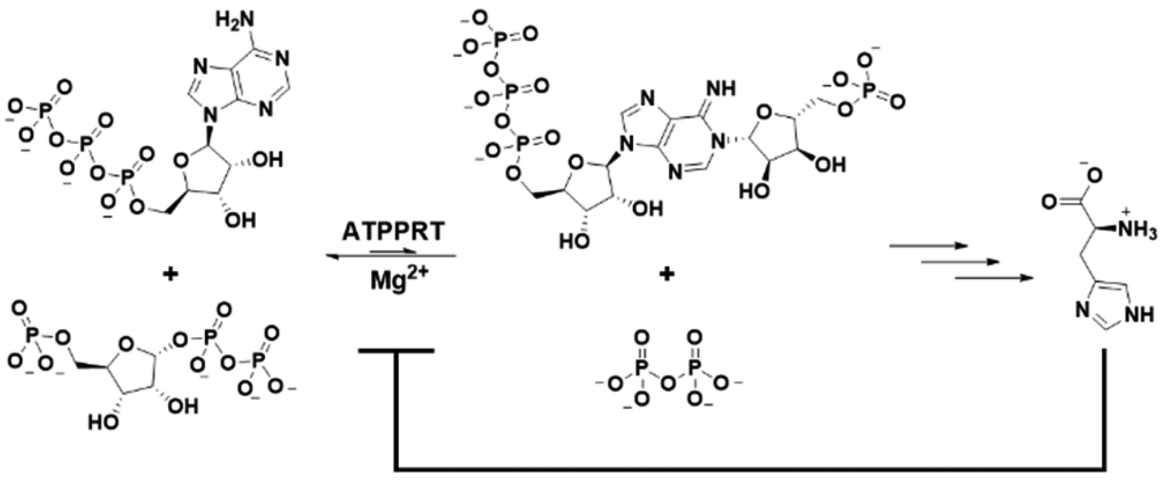

steady-state and pre-steady-state kinetics and determine the crystal structure of the PaATPPRT-PRPP-histidine complex to elucidate the mechanism of allosteric inhibition of shortform ATPPRTs by histidine.

\section{MATERIALS AND METHODS}

Materials. ATP, PRPP, adenosine 5 '-triphosphate (ADP), L-histidine, 3-(2-thienyl)-L-alanine (TIH), $\mathrm{MgCl}_{2}$, dithiothreitol (DTT), and tricine were purchased from Sigma-Aldrich. All other chemicals were purchased from readily available commercial sources. All chemicals were used without further purification. $\mathrm{PaHisG}_{\mathrm{S}}, \mathrm{PaHisZ}$, and Mycobacterium tuberculosis pyrophosphatase (MtPPase) were produced as previously published. ${ }^{20}$ The concentrations of $\mathrm{PaHisG}_{\mathrm{S}}, \mathrm{PaHisZ}$, and the $\mathrm{PaATPPRT}$ holoenzyme were calculated as previously described. $^{20}$

PaHisG $_{\mathrm{s}}$ and PaATPPRT Activity Assay. All assays were performed under initial rate conditions in the forward direction at $20^{\circ} \mathrm{C}$, in the presence or absence of histidine, as previously described $^{20}$ by monitoring the increase in absorbance at 290 $\mathrm{nm}$ due to the formation of PRATP $\left(\varepsilon_{290}=3600 \mathrm{M}^{-1} \mathrm{~cm}^{-1}\right)^{23}$ in $1 \mathrm{~cm}$ path length quartz cuvettes (Hellma) in a Shimadzu UV-2600 spectrophotometer. Unless stated otherwise, for $\mathrm{PaHisG}_{\mathrm{S}}$ activity, the $\mathrm{PaHisG}$ S concentration was typically 2 $\mu \mathrm{M}$, and for PaATPPRT activity, $\mathrm{PaHisG}_{\mathrm{S}}$ and $\mathrm{PaHisZ}$ concentrations were 0.28 and $15 \mu \mathrm{M}$, respectively. Reactions were started by addition of PRPP. Control reaction mixtures lacked either ATP, PRPP, $\mathrm{PaHisG}$, or $\mathrm{PaHisZ}$. Controls were also carried out to ensure that the rate did not depend on MtPPase. Initial rates were also measured with saturating concentrations of both substrates in the presence or absence of $4 \mathrm{mM}$ TIH. All kinetic measurements were performed at least in duplicate unless stated otherwise.

Differential Scanning Fluorimetry (DSF). DSF measurements $\left(\lambda_{\mathrm{ex}}=490 \mathrm{~nm} ; \lambda_{\mathrm{em}}=610 \mathrm{~nm}\right)$ were performed in 96well plates on a Stratagene Mx3005p instrument. Thermal denaturation of $9.4 \mu \mathrm{M} \mathrm{PaHisZ}$ was assayed $(50 \mu \mathrm{L})$ in the presence of several histidine concentrations $(0-8 \mathrm{mM})$ in 100 $\mathrm{mM}$ tricine, $100 \mathrm{mM} \mathrm{KCl}, 4 \mathrm{mM} \mathrm{DTT}$, and $15 \mathrm{mM} \mathrm{MgCl}_{2}$ ( $\mathrm{pH} 8.5$ ). Sypro Orange ( $5 \times)$ (Invitrogen) was added to all wells. Thermal denaturation curves were recorded over a temperature range of $25-65{ }^{\circ} \mathrm{C}$ with $1{ }^{\circ} \mathrm{C} \mathrm{min}{ }^{-1}$ increments. Control curves without the enzyme and were subtracted from curves with the enzyme. All measurements were carried out in quintuplicate.

PaATPPRT Saturation Kinetics with ADP and PRPP. Initial rates for PaATPPRT $(0.28 \mu \mathrm{M})$ were determined at saturating concentrations of one substrate and varying concentrations of the other, either $\mathrm{ADP}(0.4-5.6 \mathrm{mM})$ or PRPP (0.1-2.0 mM).

PaATPPRT Inhibition by Histidine. The half-maximal inhibitory concentration of histidine was determined by measuring the initial rates for PaATPPRT $(0.24 \mu \mathrm{M})$ in the presence of $5.6 \mathrm{mM}$ ATP (or $5.6 \mathrm{mM} \mathrm{ADP}$ ), $2 \mathrm{mM}$ PRPP, and varying concentrations of histidine $(0-320 \mu \mathrm{M})$. The inhibition mechanism was investigated by measuring the initial rates for PaATPPRT $(0.28 \mu \mathrm{M})$ at saturating concentrations of one substrate and varying concentrations of the other, either ATP $(0.2-2.8 \mathrm{mM})$ or PRPP $(0.1-2.0 \mathrm{mM})$, with different concentrations of histidine $(0-10 \mu \mathrm{M})$. In some instances, the lowest-concentration data point was an outlier in the doublereciprocal plot and was excluded from the fit.

Rapid Kinetics. Rapid kinetics experiments were carried out under multiple-turnover conditions by monitoring the increase in absorbance at $290 \mathrm{~nm}$ upon PRATP formation at $20{ }^{\circ} \mathrm{C}$ in an Applied Photophysics SX-20 stopped-flow spectrophotometer outfitted with a $5 \mu \mathrm{L}$ mixing cell $(0.5 \mathrm{~cm}$ path length and $0.9 \mathrm{~ms}$ dead time). In all experiments, each syringe contained $100 \mathrm{mM}$ tricine $(\mathrm{pH} 8.5), 100 \mathrm{mM} \mathrm{KCl}, 4$ mM DTT, $15 \mathrm{mM} \mathrm{MgCl}_{2}$, and $10 \mu \mathrm{M}$ MtPPase. For the approach to steady state in the PaATPPRT reaction, one syringe carried $38 \mu \mathrm{M}$ PaATPPRT, $4 \mathrm{mM}$ PRPP, and either 0 or $60 \mu \mathrm{M}$ histidine, while the other carried $7 \mathrm{mM}$ ATP and either 0 or $60 \mu \mathrm{M}$ histidine. Reaction was triggered by rapidly mixing $55 \mu \mathrm{L}$ from each syringe. The increase in absorbance was monitored in a split-time base for $1 \mathrm{~s}$, with 2500 data points collected before $0.2 \mathrm{~s}$ and 2500 in the following $0.8 \mathrm{~s}$. At least four traces were acquired for each reaction, and controls lacked PRPP. For initial rates of $\mathrm{PaATPPRT}$ and $\mathrm{PaHisG}_{\mathrm{S}}$ reactions, one syringe carried either $2-4 \mu \mathrm{M} \mathrm{PaHisG}$ or $4 \mu \mathrm{M}$ PaATPPRT, $4 \mathrm{mM}$ PRPP, and either 0 or $1.28 \mathrm{mM}$ histidine, while the other carried $11.2 \mathrm{mM}$ ATP. Reaction was triggered by rapidly mixing $55 \mu \mathrm{L}$ from each syringe. The increase in absorbance was monitored in a linear-time base for $10 \mathrm{~s}$. At least four traces were acquired for each reaction, with 1000 data points collected per trace. Controls lacked PRPP.

Protein Crystallography. PaATPPRT crystallization was performed as previously described. ${ }^{20}$ Crystals were washed in a buffer containing $10 \%$ polyethylene glycol 3350, $0.1 \mathrm{M}$ bicine ( $\mathrm{pH} 8.5$ ), $50 \mathrm{mM} \mathrm{MgCl}, 0.1 \mathrm{M} \mathrm{KBr}$, and 4\% 1,6-hexanediol and then soaked for $10 \mathrm{~min}$ a $1 \mu \mathrm{L}$ drop of this solution in which PRPP and ATP had been dissolved, as previously reported. ${ }^{10}$ Crystals were then transferred to a fresh drop of the soaking solution substituted with histidine and $20 \% 2$ methyl-2,4-pentanediol for $20 \mathrm{~s}$ and flash-cooled in a stream of nitrogen gas at $100 \mathrm{~K}$. Data were collected at beamline i24 at 

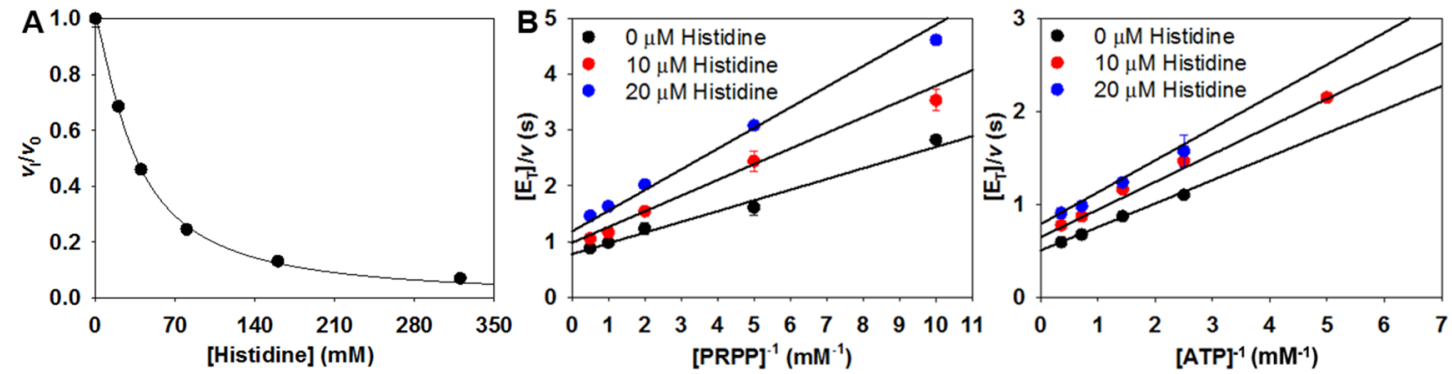

Figure 1. PaATPPRT inhibition by histidine. (A) Dose-response curve for histidine concentration. The line is the best fit of the data to eq 2. (B) Double-reciprocal plots of substrate saturation curves in the presence of histidine. Lines are reciprocals of the best fit of the data to eq 5 .

the Diamond Light Source (Oxfordshire, U.K.) and processed using the automated processing pipeline integrated with XDS. $^{24}$ The data were scaled using AIMLESS, ${ }^{25}$ and the structure was determined with MOLREP ${ }^{26}$ using Protein Data Bank (PDB) entry $6 \mathrm{FTT}^{10}$ as a search model. The model, showing the full hetero-octamer in the asymmetric unit, was refined using cycles of model building with $\mathrm{COOT}^{27}$ and refinement with REFMAC. ${ }^{28}$ Electron density in the omit map showed the presence of histidine in the four $\mathrm{PaHisZ}$ subunits and PRPP in the four $\mathrm{PaHisG}_{\mathrm{S}}$ ones. Some electron density was seen in one of the $P a H i s G_{S}$ chains where in previous structures the adenine ring of ATP was observed; however, it could not be unambiguously modeled and was left empty. The final coordinates were deposited in the Protein Data Bank (entry 6R02).

Site-Directed Mutagenesis of PaHisZ. Replacement of tyrosine for phenylalanine at position 263 of $\mathrm{PaHisZ}$ was accomplished by the method of Liu and Naismith, ${ }^{29}$ with primers 5'-GGCTTCCACTACCATACGGGTATTGTTTTCAACGGTTATATC-3' and 5'-CGTATGGTAGTGGAAGCCGCTCAATTCGGTAACGTCAATG-3'. Successful mutation was confirmed by DNA sequencing (Eurofins Genomics). Y263F-PaHisZ was expressed and purified by the same protocol reported for wild-type (WT) $\mathrm{PaHisZ}{ }^{20} \mathrm{ESI} / \mathrm{MS}$ analysis of the protein yielded a mass of 43068.9, exactly what would be expected by the loss of a hydroxyl group when compared with the mass of WT-PaHisZ. ${ }^{20}$ Y263F-PaHisZbased PaATPPRT was assayed for catalytic activity under initial rate conditions at $20{ }^{\circ} \mathrm{C}$ in the presence of $5.6 \mathrm{mM}$ ATP and $2 \mathrm{mM}$ PRPP and in the presence or absence of $320 \mu \mathrm{M}$ histidine, along with other reaction components exactly as described for WT-PaATPPRT. Measurements were carried out in duplicate.

Analysis of Kinetic and Thermal Denaturation Data. Kinetic and DSF data were analyzed by the nonlinear regression function of SigmaPlot 13 (SPSS Inc.). Data points and error bars in graphs are represented as means \pm the standard error, and kinetic and equilibrium constants are presented as means \pm the fitting error. Substrate saturation data were fitted to eq 1 . Inhibition data at fixed substrate concentrations were fitted to eq 2 , and inhibition mechanism data were fitted to eq 3 . In eqs $1-3, v$ is the initial rate, $S$ is the concentration of the varying substrate, $k_{\mathrm{cat}}$ is the steady-state turnover number, $K_{\mathrm{M}}$ is the apparent Michaelis constant, $E_{\mathrm{T}}$ is total enzyme concentration, $v_{\mathrm{i}}$ is the initial rate in the presence of an inhibitor, $\mathrm{IC}_{50}$ is the half-maximal inhibitory concentration, $h$ is the Hill slope, $K_{\text {is }}$ is the slope inhibition constant, and $K_{\mathrm{ii}}$ is the intercept inhibition constant. DSF thermal denaturation data were fitted to eq $4,{ }^{30}$ and $\mathrm{PaHisZ}$-histidine complex equilibrium dissociation data were fitted to eq 5 . In eqs 4 and $5, F_{U}$ is the fraction unfolded, $T$ is the temperature in degrees Celsius, $T_{\mathrm{m}}$ is the melting temperature, $c$ is the slope of the transition region, LL and UL are folded and unfolded baselines, respectively, $T_{\mathrm{m} 0}$ is the $T_{\mathrm{m}}$ in the absence of histidine, $T_{\mathrm{m} \infty}$ is the $T_{\mathrm{m}}$ at an infinite histidine concentration, $H$ is the histidine concentration, and $K_{\mathrm{D}}$ is the apparent equilibrium dissociation constant for the $\mathrm{PaHisZ}$-histidine complex.

$$
\begin{aligned}
\frac{v}{E_{\mathrm{T}}} & =\frac{k_{\mathrm{cat}} S}{K_{\mathrm{M}}+S} \\
\frac{v_{\mathrm{i}}}{v} & =\frac{1}{1+\left(\frac{I}{\mathrm{IC}_{\mathrm{s} 0}}\right)^{h}} \\
\frac{v}{E_{\mathrm{T}}} & =\frac{k_{\mathrm{cat}} S}{\left(1+I / K_{\mathrm{is}}\right) K_{\mathrm{M}}+\left(1+I / K_{\mathrm{ii}}\right) S} \\
F_{\mathrm{U}} & =\mathrm{LL}+\frac{\mathrm{UL}-\mathrm{LL}}{1+\mathrm{e}^{\left(T_{\mathrm{m}}-T\right) / c}} \\
T_{\mathrm{m}} & =T_{\mathrm{m} 0}+\frac{T_{\mathrm{m} \infty} H}{K_{\mathrm{D}}+H}
\end{aligned}
$$

\section{RESULTS AND DISCUSSION}

Histidine Is a Noncompetitive Inhibitor of PaATPPRT. Histidine inhibits PaATPPRT with an $\mathrm{IC}_{50}$ of $35.5 \pm 0.8 \mu \mathrm{M}$ and an $h$ of $1.30 \pm 0.04$ (Figure 1A), both values being in close agreement with those reported for the inhibition of $M$. tuberculosis $\mathrm{HisG}_{\mathrm{L}}$ ATPPRT. ${ }^{17}$ Inhibition is noncompetitive against both substrates (Figure 1B), and data fitted to eq 3 yielded a $K_{\mathrm{is}}$ of $22 \pm 6 \mu \mathrm{M}$ and a $K_{\mathrm{ii}}$ of $38 \pm 7 \mu \mathrm{M}$ against PRPP and a $K_{\text {is }}$ of $56 \pm 25 \mu \mathrm{M}$ and a $K_{\mathrm{ii}}$ of $36 \pm 5 \mu \mathrm{M}$ against ATP. Each double-reciprocal curve was also fitted individually, and linear regression of the slope and intercept replots (Figure S1) produced a $K_{\text {is }}$ of $30 \mu \mathrm{M}$ and a $K_{\mathrm{ii}}$ of $26 \mu \mathrm{M}$ against PRPP and a $K_{\text {is }}$ of $60 \mu \mathrm{M}$ and a $K_{\text {ii }}$ of $40 \mu \mathrm{M}$ against ATP, in reasonable agreement with those obtained from a global fit to eq 3 .

Histidine is a noncompetitive inhibitor of other HisG $_{S}$ ATPPRTs $^{22,31}$ and of HisG ATPPRTs, $^{3,16}$ except that from $M$. tuberculosis ATPPRT, where inhibition is uncompetitive against ATP. ${ }^{17}$ The $K_{\mathrm{i}}$ values for PaATPPRT are similar to those reported for Campylobacter jejuni, ${ }^{16}$ M. tuberculosis, ${ }^{17}$ and Lactococcus lactis ${ }^{31}$ ATPPRTs but nearly an order of magnitude lower than those reported for the Salmonella typhimurium ${ }^{3}$ and T. maritima ${ }^{22}$ enzymes. 

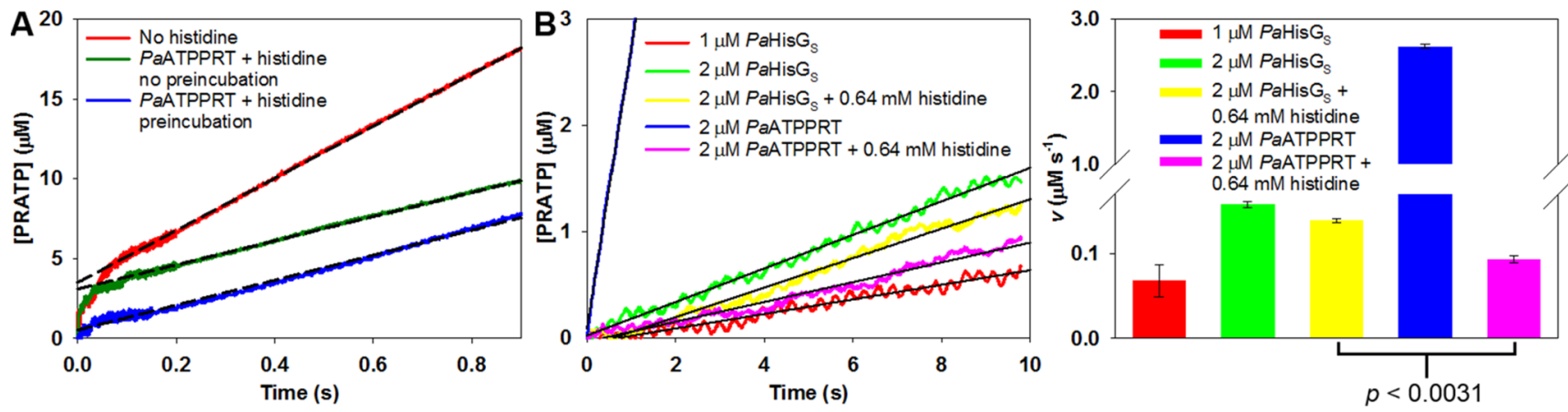

Figure 2. Rapid kinetics of histidine inhibition. (A) Effect of histidine-enzyme preincubation on the burst in product formation in the PaATPPRT reaction. Dashed lines show the linear regression of the steady-state phase, and burst amplitudes are extrapolated from the $y$-axis intercepts. (B) Time course of product formation from activated and non-activated PaATPPRT rapid kinetics in the presence and absence of histidine (left), where black lines show the linear regression of the data. The bar graph (right) represents the initial rates extracted from the time courses.

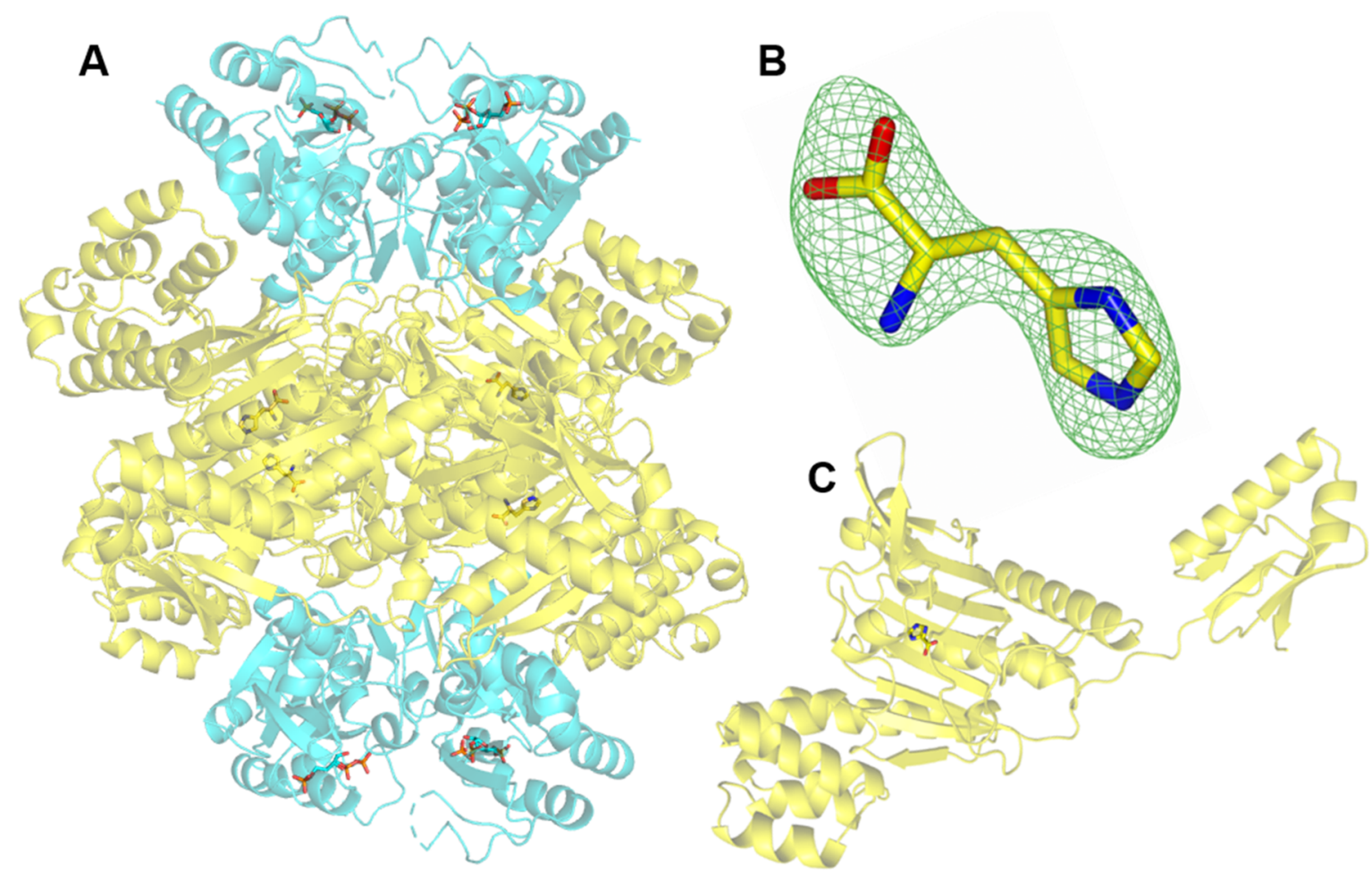

Figure 3. Crystal structure of the PaATPPRT-PRPP-histidine complex. (A) Ribbon diagram of the PaATPPRT hetero-octamer found in the asymmetric unit. (B) Omit map at $3 \sigma$ showing electron density for histidine. (C) Ribbon diagram of the PaHisZ subunit with the location of the bound histidine. $\mathrm{PaHisG}_{\mathrm{S}}$ subunits are colored cyan, and $\mathrm{PaHisZ}$ is colored yellow. Ligands are shown as sticks with carbon atoms matching the color of the subunits to which they are bound.

Low-Affinity Binding of Histidine to Free PaHisZ. The binding of the inhibitor to free PaHisZ was tested by DSF. Histidine increases the $T_{\mathrm{m}}$ of $\mathrm{PaHisZ}$ (Figure S2A), but the apparent affinity of the inhibitor for the free regulatory subunit is low (Figure S2B), with a $K_{\mathrm{D}}$ of $2.8 \pm 0.8 \mathrm{mM}$. This indicates that $\mathrm{PaHisG}_{\mathrm{S}}$ is not strictly necessary for the binding of histidine to $\mathrm{PaHisZ}$, though the hetero-octameric arrangement may be necessary to increase the affinity of the interaction.

Catalysis and Inhibition with ADP as the Substrate. ADP has been shown to be a substrate, replacing ATP, for non-activated $\mathrm{PaHisG}_{\mathrm{S}},{ }^{11}$ and the crystal structure of the PaATPPRT-PRPP-ADP complex suggests the same may be true for the activated enzyme. ${ }^{10}$ This hypothesis is confirmed here, with ADP efficiently replacing ATP as a substrate for PaATPPRT (Figure S3A) with a $k_{\text {cat }}$ of $2.6 \pm 0.4 \mathrm{~s}^{-1}$, a $k_{\text {cat }} /$
$K_{\mathrm{M}}{ }^{\text {PRPP }}$ of $(3.8 \pm 0.4) \times 10^{3} \mathrm{M}^{-1} \mathrm{~s}^{-1}$, and a $k_{\text {cat }} / K_{\mathrm{M}}{ }^{\mathrm{ADP}}$ of $(1.6$ $\pm 0.5) \times 10^{3} \mathrm{M}^{-1} \mathrm{~s}^{-1}$, in ranges similar to those obtained with ATP. $^{20}$ In addition, histidine can inhibit the PaATPPRT reaction with $\mathrm{ADP}$ as the substrate (Figure $\mathrm{S} 3 \mathrm{~B}$ ) with an $\mathrm{IC}_{50}$ of $31 \pm 1 \mu \mathrm{M}$ and an $h$ of $1.32 \pm 0.09$, in striking agreement with the results for the reaction with ATP. This indicates that neither catalysis nor allosteric inhibition involves the interaction between $\mathrm{Arg} 73$ of $\mathrm{PaHisG}_{\mathrm{S}}$ and the $\gamma$ - $\mathrm{PO}^{4-}$ group of ATP, which is absent when ADP replaces ATP. ${ }^{10}$

Effect of Histidine on the Approach to the Steady State. PaATPPRT catalysis follows an ordered mechanism in which PRPP binds first to the enzyme and involves a burst of on-enzyme PRATP formation in the first turnover followed by a lower steady-state rate dominated by product release. ${ }^{11}$ When ATP and histidine are rapidly mixed with PaATPPRT 
preincubated with PRPP (Figure 2A), the amplitude of the burst phase, whose values are $3.49 \pm 0.01$ and $3.11 \pm 0.01 \mu \mathrm{M}$ in the presence and absence of histidine, respectively, is unchanged within experimental error. The subsequent steadystate rates, whose values are $16.36 \pm 0.01$ and $7.56 \pm 0.01 \mu \mathrm{M}$ $\mathrm{s}^{-1}$ in the absence and presence of histidine, respectively, reflect the inhibition. This suggests the rates of binding of ATP to the PaATPPRT-PRPP complex and the subsequent onenzyme product formation are higher than the rate of histidine binding and establishment of inhibition. Conversely, when ATP is rapidly mixed with $P a A T P P R T$ preincubated with PRPP and histidine (Figure 2A), the steady-state rate $(7.83 \pm$ $0.01 \mu \mathrm{M} \mathrm{s}^{-1}$ ) shows the same level of inhibition but the burst amplitude is drastically reduced to $0.53 \pm 0.01 \mu \mathrm{M}$, confirming that histidine can establish an equilibrium with the PaATPPRT-PRPP binary complex to inhibit the reaction before ATP binds to trigger the first turnover. This contrasts with the case for M. tuberculosis ATPPRT, in which the burst amplitude decreases when ATP and histidine are rapidly mixed with the enzyme. $^{17}$

Histidine Does Not Lead to Dissociation of PaHisG from PaHisZ. $\mathrm{PaHisG}_{\mathrm{S}}$ is catalytically active, albeit with reduced activity in the absence of $\mathrm{PaHisZ}$, while being insensitive to histidine. ${ }^{20}$ Thus, the possibility that allosteric inhibition is a result of dissociation of $\mathrm{PaHis}_{\mathrm{S}}$ from the PaATPPRT holoenzyme was considered, and the background rate in the presence of histidine was that of the non-activated $\mathrm{PaHisG}_{\mathrm{S}}$. This was interrogated by comparing the initial rates of activated and non-activated $\mathrm{PaHisG}_{\mathrm{S}}$ at the same concentration in the presence and absence of histidine (Figure 2B). The rate of non-activated $\mathrm{PaHisG}_{\mathrm{S}}$ in the presence of histidine was shown by a Student's $t$ test to be higher than the rate of PaATPPRT in the presence of histidine at the $p<$ 0.0031 level. This indicates that allosteric inhibition by histidine does not result from release of $\mathrm{PaHisG}_{\mathrm{S}}$ from $P a A T P P R T$ to yield the non-activated free $P a H i s G_{S}$ rate.

Crystal Structure of the PaATPPRT-PRPP-Histidine Complex. To gain insight into the structural underpinnings of PaATPPRT allosteric inhibition, the $2.65 \AA$ resolution crystal structure of the PaATPPRT-PRPP-histidine complex was determined, and refinement statistics are listed in Table S1. The PaATPPRT-PRPP-histidine complex crystallized in space group $P 2_{1}$ with a full hetero-octamer in the asymmetric unit. Each $\mathrm{PaHisG}$ solecule contained a PRRP molecule bound in the active site in a similar arrangement as previously found (Figure 3A). ${ }^{10}$ The omit map shows clear electron density for one molecule of histidine (Figure 3B) bound along the central antiparallel $\beta$-sheet of each molecule of $\mathrm{PaHisZ}$ (Figure 3C), more than $18 \AA$ from the nearest $\mathrm{PaHisZ}-$ $\mathrm{PaHisG}_{\mathrm{S}}$ interface. No electron density for potential ligands was found in the $\mathrm{PaHis} \mathrm{G}_{\mathrm{S}}-\mathrm{PaHisZ}$ interface.

The structure of the PaATPPRT-PRPP-histidine complex is in sharp contrast with the only other available structure of a $\mathrm{HisG}_{\mathrm{S}}$ ATPPRT bound to histidine, that from T. maritima, in which two molecules of histidine per molecule of HisZ are reported, both in the interface between the catalytic and regulatory subunits. ${ }^{22}$ An overlay of the histidine-bound $\mathrm{PaHisZ}$ structure described here with that of $T$. maritima and the histidine-bound HisRS from Burkholderia thailandensis $^{32}$ (Figure S4) highlights the striking differences in the location of the histidine-binding sites between the two HisZ proteins. Interestingly, the binding position of histidine is almost identical in $\mathrm{PaHisZ}$ and HisRS. The proposal that
HisRS and HisZ would share a similar location for the histidine-binding site had been previously suggested, ${ }^{21}$ which is now demonstrated by the current structure.

Histidine-Binding Site. The histidine-binding site is formed solely by $\mathrm{PaHisZ}$ residues. The inhibitor makes several polar interactions with the side chains of eight $\mathrm{PaHisZ}$ residues (Figure 4). The $\alpha$ - $\mathrm{COO}^{-}$group of histidine contacts the $\delta$-NH

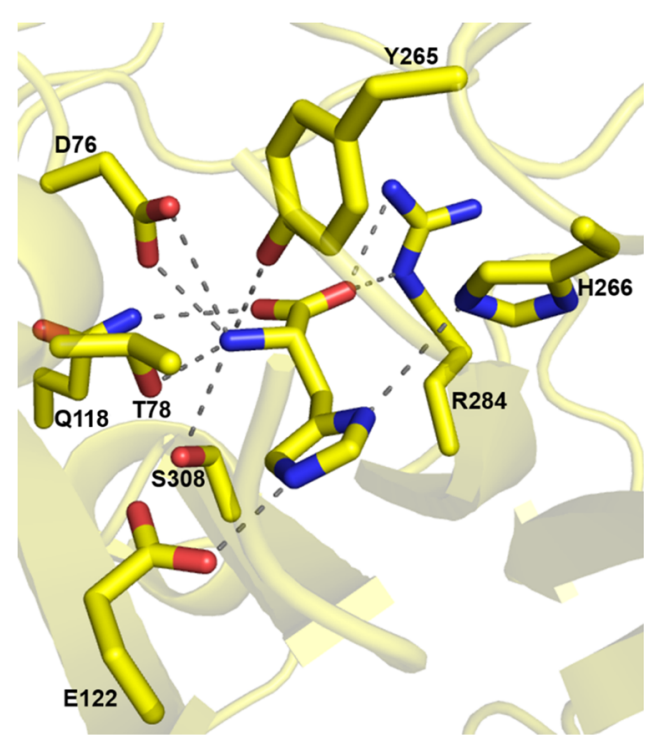

Figure 4. Close-up of the histidine-binding site in PaHisZ. The inhibitor and the relevant side chains are depicted as sticks, with carbons colored yellow, nitrogens blue, and oxygens red. The dashed lines represent polar contacts.

and $\omega-\mathrm{NH}_{2}$ groups of $\operatorname{Arg} 284$ and the $\gamma-\mathrm{NH}_{2}$ group of Gln118. The histidine $\alpha-\mathrm{NH}_{3}{ }^{+}$group interacts via hydrogen bonds with the Tyr265 4-OH group and Ser308 and Thr78 $\beta$-OH groups and via a salt bridge with the Asp76 $\beta-\mathrm{COO}^{-}$group. The imidazole ring of the inhibitor is anchored to the $\beta$-strand via an interaction between its $\tau$-NH and Glu122 $\gamma$-COO ${ }^{-}$and further contacts a flexible loop that also includes Tyr265 via an interaction between its $\pi$-N and His266 $\tau$-NH. The interactions with the imidazole ring of the inhibitor must be essential for allosteric inhibition, because TIH, a histidine analogue expected to bind in a manner similar to that of histidine but whose five-membered ring does not participate in polar interactions, ${ }^{9}$ has no effect on PaATPPRT catalysis (Figure S5). Interestingly, most $\mathrm{PaHisZ}$ residues responsible for polar contacts with the allosteric inhibitor are conserved in T. maritima HisZ (Figure S6), even though histidine is not reported to bind to that site. Two of the residues, Tyr265 and His 266, are replaced by glutamate and tyrosine, respectively, in T. maritima HisZ, which would still be able to make similar interactions as seen in $\mathrm{PaHisZ}$. The only significant exception is Arg284, which has no replacement capable of polar contacts in the T. maritima protein.

Structural Basis for PaATPPRT Allosteric Inhibition. $\mathrm{HisG}_{\mathrm{S}}$ ATPPRTs are intriguing hetero-octameric allosteric systems in which HisZ mediates allosteric activation and inhibition of catalysis by $\mathrm{HisG}_{\mathrm{S}}$, depending on the presence of histidine. $^{6,8,20}$ The mechanism of PaATPPRT allosteric activation has recently been uncovered and involves the subunits of the $\mathrm{PaHis} \mathrm{G}_{\mathrm{S}}$ dimer moving closer to one another, leading to a change in the average position of a $\mathrm{PaHis}_{\mathrm{S}}$ loop that reaches across the adjacent subunit to provide leaving 

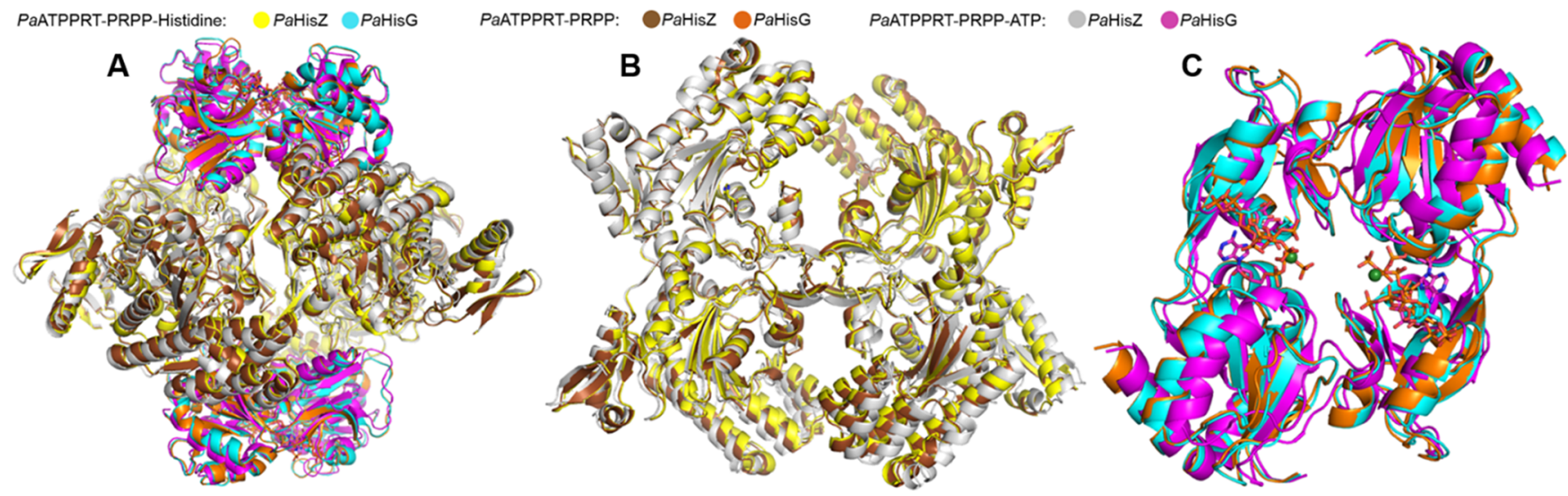

Figure 5. Overlay of the structures of PaATPPRT-PRPP, PaATPPRT-PRPP-histidine, and PaATPPRT-PRPP-ATP complexes. (A) Overlay of the full hetero-octamers. (B) Overlay of the PaHisZ tetramers. (C) Overlay of the $\mathrm{PaHisG}_{\mathrm{S}}$ dimers.

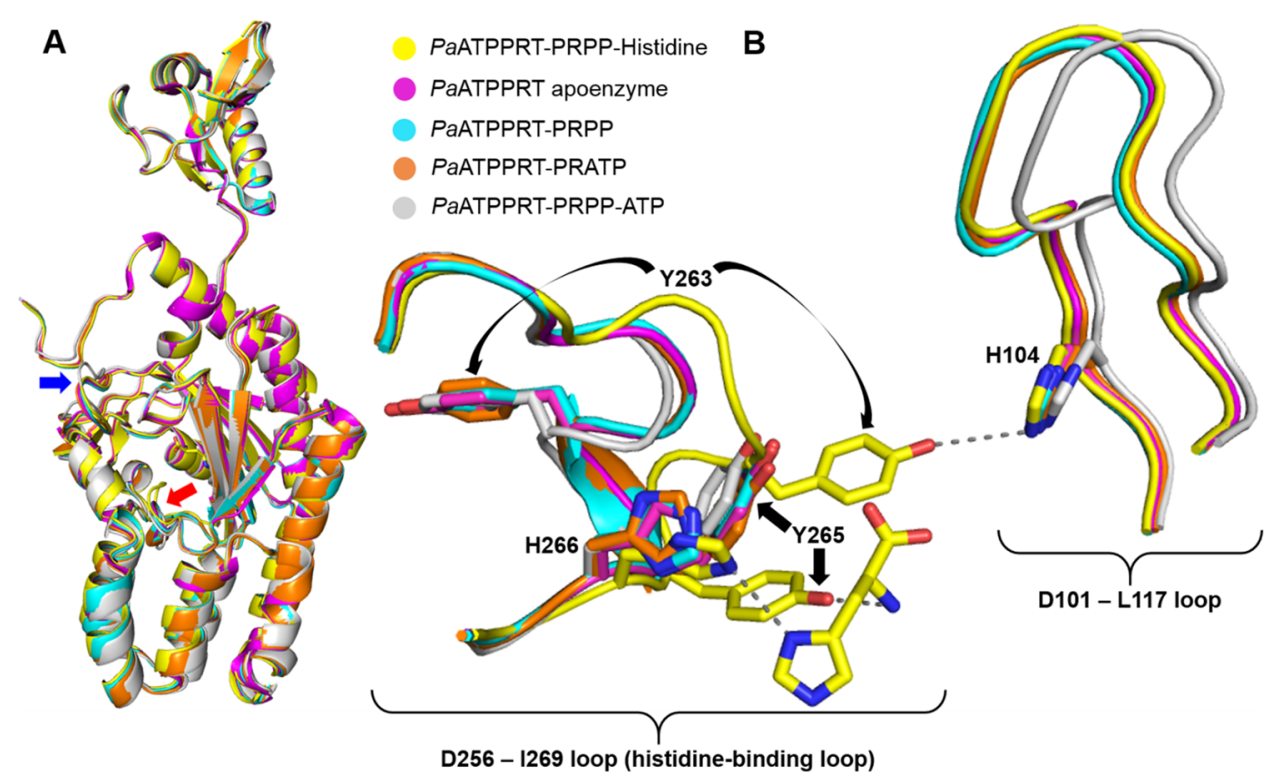

Figure 6. PaHisZ subunit conformations across PaATPPRT structures. (A) Overlay of PaHisZ subunits from all PaATPPRT structures. The red arrow points to the histidine-binding loop, and the blue arrow points to the Asp101-Leu117 loop. (B) Close-up view of the two loops, key side chains, and the inhibitor histidine. PaHisZ residue side chains and bound histidine are shown as sticks. Dashed lines denote polar interactions. Black arrows highlight the different positions adopted by the same side chains in the different structures.

group stabilization at the transition state via a salt bridge between its Arg56 and the PRPP PP moiety. $^{10,11}$ However, this is only manifested upon binding of ATP to the PaATPPRT-PRPP binary complex, as all other structures of $P a A T P P R T$, namely, the apoenzyme and the binary complexes with PRPP and PRATP, are in a non-activated state. ${ }^{10}$ An overlay of the crystal structures of the PaATPPRT-PRPP, PaATPPRT-PRPP-histidine, and PaATPPRT-PRPP-ATP (Figure 5A) complexes resulted in $\mathrm{C} \alpha$ root-mean-square deviations (rmsds) of $0.40 \AA$ for the first two structures but $1.71 \AA$ for the latter two. When the $\mathrm{PaHisZ}$ tetramers are overlaid (Figure 5B), an rmsd of $0.38 \AA$ resulted for the first two structures and an rmsd of $1.37 \AA$ for the latter two. An overlay of the $P a$ His $G_{S}$ dimers (Figure $5 \mathrm{C}$ ) yielded an rmsd of $0.27 \AA$ for the first two structures and $1.75 \AA$ for the latter two. These results suggest that both PaATPPRT-PRPP and PaATPPRT-PRPP-histidine complexes are in an overall very similar non-activated conformation. However, the PaATPPRT-PRPP complex is en route to activation upon ATP binding, but histidine binding may lock the hetero- octamer in a non-activated state, preventing the conformational changes that would otherwise take place following ATP binding.

A Potential Structural Path for Allosteric Inhibition. In an attempt to uncover how the structural rearrangements that may prevent activation of the PaATPPRT-PRPPhistidine complex are relayed from the inhibitor-binding site to the active site, $\mathrm{PaHisZ}$ subunits from each structure (PaATPPRT, ${ }^{20}$ PaATPPRT-PRPP, PaATPPRT-PRPPATP, PaATPPRT-PRATP, ${ }^{10}$ and PaATPPRT-PRPP-histidine) were overlaid over their $\mathrm{C} \alpha$ atoms (Figure 6A). The conformations of the $\mathrm{PaHisZ}$ monomers are very similar across all structures, except for the position of two loops. The first is the histidine-binding loop (red arrow in Figure 6A), spanning residues Asp256-Ile269 (Figure 6B), in the PaATPPRTPRPP-histidine complex, which moves $>4.5 \AA$ from its position in all other structures (Figure $6 \mathrm{~B}$ ). This loop includes His266, whose side chain adopts a slightly different rotamer to interact with the inhibitor, and $\mathrm{Tyr} 265$, whose 4-OH group is $>6 \AA$ from its position in the other structures, forming a 


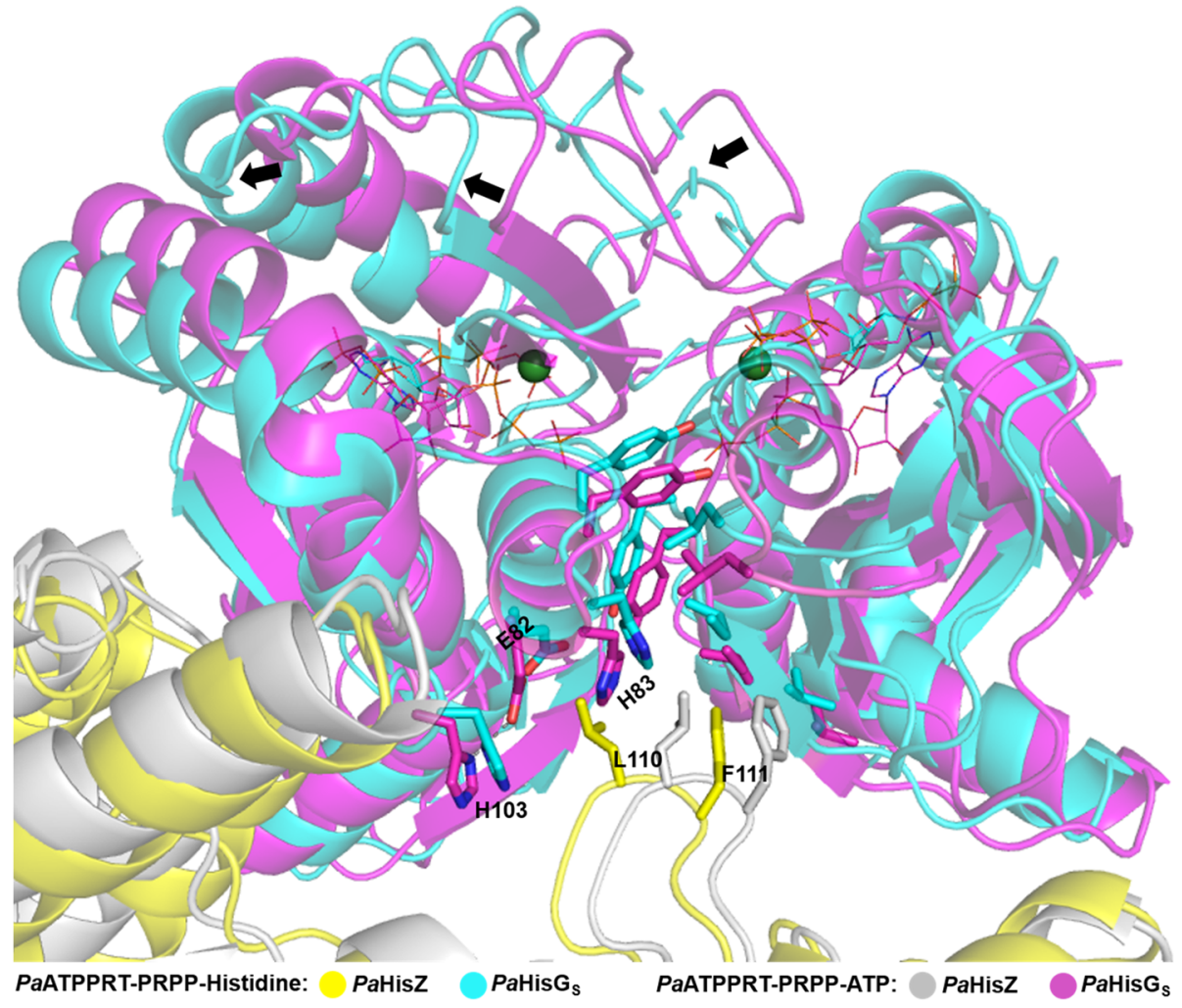

Figure 7. Ribbon diagram of the $\mathrm{PaHisZ}-\mathrm{PaHis} \mathrm{S}_{\mathrm{S}}$ and $\mathrm{PaHis} \mathrm{S}_{\mathrm{S}}-\mathrm{PaHisG}$ interface in overlaid structures of $\mathrm{PaATPPRT}-\mathrm{PRPP}-\mathrm{histidine}$ and $\mathrm{PaATPPRT}-\mathrm{PRPP}-\mathrm{ATP}$ complexes. For the sake of clarity, only part of one $\mathrm{PaHisZ}$ and one of the $\mathrm{PaHis} \mathrm{G}_{\mathrm{S}}$ dimers are shown for each structure. Side chains are shown as sticks, and substrates as wireframe. The $\mathrm{Mg}^{2+}$ ions in the structure of the PaATPPRT-PRPP-ATP complex are represented as green spheres. Black arrows denote the distinct positions of the $\mathrm{PaHis}_{\mathrm{S}}$ loop responsible for leaving group stabilization in the transition state.

hydrogen bond with the bound histidine (Figure 6B, yellow structure). The second (blue arrow in Figure $6 \mathrm{~A}$ ) is the loop spanning residues Asp101-Leu117 (Figure 6B), which in the structure of the activated PaATPPRT-PRPP-ATP complex is $>2 \AA$ from its position in all other structures (Figure 6B, gray structure). These two loops are connected in the structure of the PaATPPRT-PRPP-histidine complex via a hydrogen bond between Tyr263 in the histidine-binding loop, which has rotated almost $180^{\circ}$ from its position in all other structures, and His104 in the Asp101-Leu117 loop (Figure 6B). This interaction is missing from all other $\mathrm{PaATPPRT}$ structures.

The Asp101-Leu117 loop leads directly to the PaHisZ$\mathrm{PaHis}_{\mathrm{S}}$ interface and is poised for hydrophobic interactions via the side chains of its Leu110 and Phe111 residues with one of the $\mathrm{PaHisG}_{\mathrm{S}}$ monomers in the activated PaATPPRTPRPP-ATP complex (Figure 7, PaHisZ colored gray and $\mathrm{PaHisG}_{\mathrm{S}}$ colored magenta). Nonetheless, in the allosterically inhibited PaATPPRT-PRPP-histidine complex, the position of this loop is shifted toward the other $\mathrm{PaHisG}_{\mathrm{S}}$ monomer (Figure 7, $\mathrm{PaHisZ}$ colored yellow and $\mathrm{PaHisG}$ s colored cyan), with $\mathrm{PaHisZ}$ Leu110 disrupting a Glu82-His103 interaction in $\mathrm{PaHisG}_{\mathrm{S}}$. Furthermore, the aromatic ring of $\mathrm{PaHisZ}$ Phe111 occupies a space closer to the interface between the two $\mathrm{PaHisG}_{\mathrm{S}}$ subunits (Figure 7, PaHisZ colored yellow and $\mathrm{PaHisG}_{\mathrm{S}}$ colored cyan), further perturbing the hydrophobic interactions present in the activated complex (Figure 7, $\mathrm{PaHisZ}$ colored gray and $\mathrm{PaHisG}_{\mathrm{S}}$ colored magenta). This seems to have a knock-on effect on several other residues along the $\mathrm{PaHis} \mathrm{H}_{\mathrm{S}}-\mathrm{PaHisG}$ interface (Figure 7, magenta and cyan), which culminates in the $\alpha$-helix and $\beta$-strand connecting the loop responsible for cross-subunit stabilization of leaving group departure receding (Figure 7, cyan, indicated by black arrows) from their respective positions in the activated Michaelis complex (Figure 7, magenta) and consequently being farther from the adjacent catalytic subunit. This might be responsible, in whole or in part, for the allosteric inhibition triggered by histidine binding.

Amino acid multiple-sequence alignment of HisZs (Figure S7) shows that Tyr263 is conserved in five sequences, and His 104 in six, of 13 aligned. In two other sequences, they are replaced by residues capable of similar polar interactions. To test the hypothesis about the importance of the Tyr263His 104 interaction, the Y263F mutation was introduced into PaHisZ (Y263F-PaATPPRT). Tyr263 is not part of the histidine-binding site and as such should not impact histidine binding, but the absence of the Tyr263 4-OH group will prevent the hydrogen bond between Tyr263 and His104 from forming. If this interaction is crucial for relaying the allosteric signal to the active site, then Y263F-PaATPPRT should be insensitive to inhibition by histidine. When the enzyme activity of Y263F-PaATPPRT is measured in the absence and presence of histidine (Figure S8), significant but not complete inhibition is observed. The ratio of initial rates in the presence $\left(v_{\mathrm{i}}\right)$ and absence $\left(v_{0}\right)$ of inhibitor $\left(v_{\mathrm{i}} / v_{0}\right)$ is $0.250 \pm 0.001$ for Y263FPaATPPRT, while $v_{\mathrm{i}} / v_{0}=0.070 \pm 0.004$ for WT-PaATPPRT. This is consistent with the disruption of the Tyr263-His104 hydrogen bond rendering Y263F-PaATPPRT >3-fold less susceptible than WT-PaATPPRT to inhibition by histidine, raising the possibility the interaction plays a role in mediating allostery. However, $75 \%$ of the enzyme activity of the mutant is 
still lost due to histidine inhibition, indicating that other factors are involved in the transmission of the allosteric signal.

These elusive factors may involve additional conformational changes triggered by histidine binding not evident in this structure. It is worth noting that crystals of the PaATPPRTPRPP-histidine complex could be obtained only by soaking, which might hinder some conformational changes due to crystal packing. Furthermore, instead of a relatively stable conformational change, a significant portion of the allosteric signal relay may involve a transient shift in the dynamic landscape sampled by the enzyme, which would be unlikely to be captured in crystallo. Further studies, probably encompassing experimental and computational approaches, will be required for a complete elucidation of the allosteric inhibition pathway in short-form ATPPRTs.

\section{ASSOCIATED CONTENT}

\section{S Supporting Information}

The Supporting Information is available free of charge on the ACS Publications website at DOI: 10.1021/acs.biochem.9b00282.

Figures S1-S8 and Table S1 (PDF)

\section{Accession Codes}

PaHisG ${ }_{\mathrm{S}}$, UniProt Q4FQF7; PaHisZ, UniProt Q4FTX3.

\section{AUTHOR INFORMATION}

\section{Corresponding Author}

*E-mail: rgds@st-andrews.ac.uk. Phone: +44 01334463496.

\section{ORCID}

Rafael G. da Silva: 0000-0002-1308-8190

\section{Author Contributions}

${ }^{\dagger}$ C.M.T. and M.S.A. contributed equally to this work.

\section{Funding}

This work was supported by a grant from the Wellcome Trust Institutional Strategic Support Fund to the University of St Andrews and the Biotechnology and Biological Sciences Research Council (BBSRC) (Grant BB/M010996/1) via an EASTBIO Doctoral Training Partnership studentship to G.F.

\section{Notes}

The authors declare no competing financial interest.

\section{ACKNOWLEDGMENTS}

X-ray diffraction data were collected at Diamond Light Source in the U.K.

\section{ABBREVIATIONS}

ATP, adenosine 5'-triphosphate; ATPPRT, ATP phosphoribosyltransferase; PRPP, 5-phospho- $\alpha$-D-ribosyl-1-pyrophosphate; PRATP, $N^{1}$-(5-phospho- $\beta$-D-ribosyl)-ATP; $\mathrm{PP}_{\mathrm{i}}$, inorganic pyrophosphate; ADP, adenosine 5 -diphosphate; DTT, dithiothreitol; TIH, 3-(2-thienyl)-L-alanine; DSF, differential scanning fluorimetry; PaATPPRT, Ps. arcticus ATPPRT; $\mathrm{PaHisG}_{\mathrm{S}}, \quad$ Ps. arcticus HisG $\mathrm{H}_{\mathrm{S}}$ PaHisZ, Ps. arcticus HisZ; MtPPase, M. tuberculosis inorganic pyrophosphatase; HisRS, histidyl-tRNA synthetase.

\section{REFERENCES}

(1) Gerhart, J. C., and Pardee, A. B. (1962) The enzymology of control by feedback inhibition. J. Biol. Chem. 237, 891-896.

(2) Monod, J., Changeux, J.-P., and Jacob, F. (1963) Allosteric proteins and cellular control systems. J. Mol. Biol. 6, 306-329.
(3) Martin, R. G. (1963) The first enzyme in histidine biosynthesis: The nature of feedback inhibition by histidine. J. Biol. Chem. 238, 257-268.

(4) Ames, B. N., Martin, R. G., and Garry, B. J. (1961) The first step of histidine biosynthesis. J. Biol. Chem. 236, 2019-2026.

(5) Martin, R. G., Berberich, M. A., Ames, B. N., Davis, W. W., Goldberger, R. F., and Yourno, J. D. (1971) Enzymes and intermediates of histidine biosynthesis in salmonella typhimurium. Methods Enzymol. 17, 3-44.

(6) Livingstone, E. K., Mittelstadt, G., Given, F. M., and Parker, E. J. (2016) Independent catalysis of the short form hisg from lactococcus lactis. FEBS Lett. 590, 2603-2610.

(7) Mittelstadt, G., Jiao, W., Livingstone, E. K., Moggre, G. J., Nazmi, A. R., and Parker, E. J. (2018) A dimeric catalytic core relates the short and long forms of atp-phosphoribosyltransferase. Biochem. J. $475,247-260$.

(8) Sissler, M., Delorme, C., Bond, J., Ehrlich, S. D., Renault, P., and Francklyn, C. (1999) An aminoacyl-trna synthetase paralog with a catalytic role in histidine biosynthesis. Proc. Natl. Acad. Sci. U. S. A. 96, 8985-8990.

(9) Pisco, J. P., de Chiara, C., Pacholarz, K. J., Garza-Garcia, A., Ogrodowicz, R. W., Walker, P. A., Barran, P. E., Smerdon, S. J., and de Carvalho, L. P. S. (2017) Uncoupling conformational states from activity in an allosteric enzyme. Nat. Commun. 8, 203.

(10) Alphey, M. S., Fisher, G., Ge, Y., Gould, E. R., Machado, T. G., Liu, H., Florence, G. J., Naismith, J. H., and da Silva, R. G. (2018) Catalytic and anticatalytic snapshots of a short-form atp phosphoribosyltransferase. ACS Catal. 8, 5601-5610.

(11) Fisher, G., Thomson, C. M., Stroek, R., Czekster, C. M., Hirschi, J. S., and da Silva, R. G. (2018) Allosteric activation shifts the rate-limiting step in a short-form atp phosphoribosyltransferase. Biochemistry 57, 4357-4367.

(12) Kulis-Horn, R. K., Persicke, M., and Kalinowski, J. (2014) Histidine biosynthesis, its regulation and biotechnological application in corynebacterium glutamicum. Microb. Biotechnol. 7, 5-25.

(13) Kulis-Horn, R. K., Persicke, M., and Kalinowski, J. (2015) Corynebacterium glutamicum atp-phosphoribosyl transferases suitable for 1-histidine production-strategies for the elimination of feedback inhibition. J. Biotechnol. 206, 26-37.

(14) Cho, Y., Sharma, V., and Sacchettini, J. C. (2003) Crystal structure of atp phosphoribosyltransferase from mycobacterium tuberculosis. J. Biol. Chem. 278, 8333-8339.

(15) Cho, Y., Ioerger, T. R., and Sacchettini, J. C. (2008) Discovery of novel nitrobenzothiazole inhibitors for mycobacterium tuberculosis atp phosphoribosyl transferase (hisg) through virtual screening. $J$. Med. Chem. 51, 5984-5992.

(16) Mittelstadt, G., Moggre, G. J., Panjikar, S., Nazmi, A. R., and Parker, E. J. (2016) Campylobacter jejuni adenosine triphosphate phosphoribosyltransferase is an active hexamer that is allosterically controlled by the twisting of a regulatory tail. Protein Sci. 25, 14921506.

(17) Pedreno, S., Pisco, J. P., Larrouy-Maumus, G., Kelly, G., and de Carvalho, L. P. (2012) Mechanism of feedback allosteric inhibition of atp phosphoribosyltransferase. Biochemistry 51, 8027-8038.

(18) Pacholarz, K. J., Burnley, R. J., Jowitt, T. A., Ordsmith, V., Pisco, J. P., Porrini, M., Larrouy-Maumus, G., Garlish, R. A., Taylor, R. J., de Carvalho, L. P. S., and Barran, P. E. (2017) Hybrid mass spectrometry approaches to determine how l-histidine feedback regulates the enzyzme mtatp-phosphoribosyltransferase. Structure 25, 730-738.e734.

(19) Bovee, M. L., Champagne, K. S., Demeler, B., and Francklyn, C. S. (2002) The quaternary structure of the hisz-hisg n-1-(5phosphoribosyl)-atp transferase from lactococcus lactis. Biochemistry $41,11838-11846$.

(20) Stroek, R., Ge, Y., Talbot, P. D., Glok, M. K., Bernas, K. E., Thomson, C. M., Gould, E. R., Alphey, M. S., Liu, H., Florence, G. J., Naismith, J. H., and da Silva, R. G. (2017) Kinetics and structure of a cold-adapted hetero-octameric atp phosphoribosyltransferase. Biochemistry 56, 793-803. 
(21) Champagne, K. S., Sissler, M., Larrabee, Y., Doublie, S., and Francklyn, C. S. (2005) Activation of the hetero-octameric atp phosphoribosyl transferase through subunit interface rearrangement by a trna synthetase paralog. J. Biol. Chem. 280, 34096-34104.

(22) Vega, M. C., Zou, P., Fernandez, F. J., Murphy, G. E., Sterner, R., Popov, A., and Wilmanns, M. (2005) Regulation of the heterooctameric atp phosphoribosyl transferase complex from thermotoga maritima by a trna synthetase-like subunit. Mol. Microbiol. 55, 675686.

(23) Smith, D. W., and Ames, B. N. (1965) Phosphoribosyladenosine monophosphate, an intermediate in histidine biosynthesis. J. Biol. Chem. 240, 3056-3063.

(24) Kabsch, W. (2010) Xds. Acta Crystallogr., Sect. D: Biol. Crystallogr. 66, 125-132.

(25) Evans, P. R., and Murshudov, G. N. (2013) How good are my data and what is the resolution? Acta Crystallogr., Sect. D: Biol. Crystallogr. 69, 1204-1214.

(26) Vagin, A., and Teplyakov, A. (1997) Molrep: An automated program for molecular replacement. J. Appl. Crystallogr. 30, 10221025.

(27) Emsley, P., and Cowtan, K. (2004) Coot: Model-building tools for molecular graphics. Acta Crystallogr., Sect. D: Biol. Crystallogr. 60, $2126-2132$

(28) Murshudov, G. N., Vagin, A. A., and Dodson, E. J. (1997) Refinement of macromolecular structures by the maximum-likelihood method. Acta Crystallogr., Sect. D: Biol. Crystallogr. 53, 240-255.

(29) Liu, H., and Naismith, J. H. (2008) An efficient one-step sitedirected deletion, insertion, single and multiple-site plasmid mutagenesis protocol. BMC Biotechnol. 8, 91.

(30) Niesen, F. H., Berglund, H., and Vedadi, M. (2007) The use of differential scanning fluorimetry to detect ligand interactions that promote protein stability. Nat. Protoc. 2, 2212-2221.

(31) Champagne, K. S., Piscitelli, E., and Francklyn, C. S. (2006) Substrate recognition by the hetero-octameric atp phosphoribosyltransferase from lactococcus lactis. Biochemistry 45, 14933-14943.

(32) Moen, S. O., Edwards, T. E., Dranow, D. M., Clifton, M. C., Sankaran, B., Van Voorhis, W. C., Sharma, A., Manoil, C., Staker, B. L., Myler, P. J., and Lorimer, D. D. (2017) Ligand co-crystallization of aminoacyl-trna synthetases from infectious disease organisms. Sci. Rep. 7, 223. 